\title{
Bronchiectasis and Nontuberculous Mycobacteria: It Is Not Over till It Is Over
}

\author{
Hyung-Jun Kim ${ }^{\mathrm{a}}$ Jae-Joon Yim ${ }^{\mathrm{b}, \mathrm{c}}$ \\ aDivision of Pulmonary and Critical Care Medicine, Department of Internal Medicine, Seoul National University \\ Bundang Hospital, Seongnam, South Korea; ${ }^{b}$ Division of Pulmonary and Critical Care Medicine, Department of \\ Internal Medicine, Seoul National University Hospital, Seoul, South Korea; 'Department of Internal Medicine, \\ Seoul National University College of Medicine, Seoul, South Korea
}

The incidence of nontuberculous mycobacterium pulmonary disease (NTM-PD) is increasing worldwide $[1,2]$. It is especially common among patients with chronic lung diseases such as bronchiectasis [3]. According to recent British Thoracic Society guidelines and European Respiratory Society guidelines $[4,5]$, patients diagnosed with bronchiectasis should be candidates for nontuberculous mycobacterial (NTM) screening. Such an evaluation is important because macrolides play important roles in treating both NTM-PD and bronchiectasis [5, 6]. Prescribing macrolides to prevent exacerbation in patients with bronchiectasis could cause macrolide resistance in those with undetected NTM colonization $[6,7]$. Data are needed on NTM-PD prevalence in patients with bronchiectasis because NTM-PD can affect the level of emphasis placed on NTM screening in these patients [5]. Thus, the European Multicentre Bronchiectasis Audit and Research Collaboration of the European Respiratory Society emphasizes evaluating the microbial colonization in patients with bronchiectasis as the third highest research priority [8].

Zhu et al. [9] attempted to resolve this issue by analyzing the prevalence of NTM isolation and NTM-PD by conducting a meta-analysis that included 21 studies. The pooled isolation rate for NTM in patients with bronchiectasis was $7.7 \%$ (95\% confidence interval $5.0-11.7 \%, I^{2}=$

karger@karger.com www.karger.com/res

Karger"
(C) 2021 The Author(s)

Published by S. Karger AG, Basel

This is an Open Access article licensed under the Creative Commons Attribution-NonCommercial-4.0 International License (CC BY-NC) (http://www.karger.com/Services/OpenAccessLicense), applicable to the online version of the article only. Usage and distribution for commercial purposes requires written permission.
$97.7 \%, p<0.001)$. Interestingly, these rates showed regional diversity: the rates in descending order were $50.0 \%$ in North America, 9.5\% in Asia, 7.5\% in Africa, 5.6\% in Oceania, and 5.4\% in Europe.

This meta-analysis is advantageous because it provides an updated systematic review that includes recent studies and an analysis of the regional prevalence of NTM infections among patients with bronchiectasis. Interestingly, the authors found that non-purulent sputum, weight loss, and fatigue were more common among those with isolated NTM than among those without isolated NTM. The authors also reported that Mycobacterium avium complex and $M$. abscessus complex were the most common species identified in patients with bronchiectasis.

However, the study included some of the common problems of meta-analyses. First, the included studies were heterogeneous. Although the authors conducted various subgroup analyses, considerable heterogeneity remained, thus making a solid conclusion unattainable [10]. Such heterogeneity resides in many patient characteristics and, more importantly, in each center's discordant principles for NTM screening. The methods of specimen analysis also differed: sputum culturing was performed in 15 studies, bronchoalveolar lavage fluid culturing was performed in one study, and sputum or
Correspondence to:

Jae-Joon Yim,yimjj@snu.ac.kr 
bronchial aspirates were performed in 4 studies. The authors of one study considered sputum polymerase chain reaction a valid method for NTM isolation; this method is not recommended for microbiologically diagnosing NTM-PD [8]. With this diversity among the studies, the pooled prevalence of the NTM identification should be accepted cautiously.

Furthermore, NTM isolation does not guarantee an NTM-PD diagnosis $[11,12]$. NTM is ubiquitous and prone to respiratory tract colonization and specimen contamination. Only three of the 21 studies included in this meta-analysis provided the NTM-PD prevalence, which is also problematic for drawing a conclusion.

Patients with bronchiectasis should be screened for NTM on initial evaluation, but these patients can also subsequently develop NTM-PD. Our analysis of the prospective cohort of 221 patients with bronchiectasis without NTM isolation at their initial evaluation provided some insight [13]. During the median follow-up duration of 55 months, NTM was isolated from 35 patients (15.8\%). Of those patients, 31 (14.0\%) were ultimately diagnosed with NTM-PD within a median 37-month observation. NTM-PD development was combined with worsening radiographic lesions.

The meta-analysis by Zhu et al. [9] provided a valuable summary of NTM prevalence among patients with bron- chiectasis. Nonetheless, it should be cautiously interpreted because of the significant heterogeneity across studies and the insufficient data on NTM-PD development. Until more homogenous studies are published, clinicians should take an individualized approach to patients with bronchiectasis considering regional NTM prevalence. Most importantly, clinicians should consider that NTMPD can develop later in patients with bronchiectasis but without NTM at their initial evaluation. It is not over till it is over.

\section{Conflict of Interest Statement}

The authors have no conflicts of interest to declare.

\section{Funding Sources}

This research received no specific grant from any funding agency.

\section{Author Contributions}

J.J.Y. and H.J.K. contributed to the concept, design, writing, and revision of the manuscript. Both of the authors read and approved the final manuscript.

\section{References}

1 Marras TK, Mendelson D, Marchand-Austin A, May K, Jamieson FB. Pulmonary nontuberculous mycobacterial disease, Ontario, Canada, 1998-2010. Emerg Infect Dis. 2013 Nov;19(11):1889-91.

2 Shah NM, Davidson JA, Anderson LF, Lalor MK, Kim J, Thomas HL, et al. Pulmonary Mycobacterium avium-intracellulare is the main driver of the rise in non-tuberculous mycobacteria incidence in England, Wales and Northern Ireland, 2007-2012. BMC Infect Dis. 2016 May 6;16:195.

3 Mirsaeidi M, Hadid W, Ericsoussi B, Rodgers $\mathrm{D}$, Sadikot RT. Non-tuberculous mycobacterial disease is common in patients with noncystic fibrosis bronchiectasis. Int J Infect Dis. 2013 Nov; 17(11):e1000-4.

4 Polverino E, Goeminne PC, McDonnell MJ, Aliberti S, Marshall SE, Loebinger MR, et al. European Respiratory Society guidelines for the management of adult bronchiectasis. Eur Respir J. 2017 Sep;50(3):1700629.

5 Hill AT, Sullivan AL, Chalmers JD, De Soyza A, Elborn SJ, Floto AR, et al. British Thoracic
Society Guideline for bronchiectasis in adults. Thorax. 2019 Jan;74(Suppl 1):1-69.

6 Daley CL, Iaccarino JM, Lange C, Cambau E, Wallace RJ, Andrejak C, et al. Treatment of nontuberculous mycobacterial pulmonary disease: an official ATS/ERS/ESCMID/IDSA clinical practice guideline: executive summary. Clin Infect Dis. 2020 Aug 14;71(4):e1-36.

7 Chaisson RE, Benson CA, Dube MP, Heifets LB, Korvick JA, Elkin S, et al. Clarithromycin therapy for bacteremic Mycobacterium avium complex disease. A randomized, doubleblind, dose-ranging study in patients with AIDS. AIDS Clinical Trials Group Protocol 157 Study Team. Ann Intern Med. 1994 Dec 15;121(12):905-11.

8 Aliberti S, Masefield S, Polverino E, De Soyza A, Loebinger MR, Menendez R, et al. Research priorities in bronchiectasis: a consensus statement from the EMBARC Clinical Research Collaboration. Eur Respir J. 2016 Sep; 48(3):632-47.

9 Zhu Y, Xie J, He X, Peng B, Wang C, Zhang $G$, et al. Prevalence and clinical characteristics of non-tuberculous mycobacteria in patients with bronchiectasis: a systematic review and meta-analysis. Respiration. 2021. Epub ahead of print.

10 Higgins JP, Thompson SG. Quantifying heterogeneity in a meta-analysis. Stat Med. 2002 Jun 15;21(11):1539-58.

11 Griffith DE, Aksamit T, Brown-Elliott BA, Catanzaro A, Daley C, Gordin F, et al. An official ATS/IDSA statement: diagnosis, treatment, and prevention of nontuberculous mycobacterial diseases. Am J Respir Crit Care Med. 2007 Feb 15;175(4):367-416.

12 Haworth CS, Banks J, Capstick T, Fisher AJ, Gorsuch T, Laurenson IF, et al. British Thoracic Society guidelines for the management of non-tuberculous mycobacterial pulmonary disease (NTM-PD). Thorax. 2017 Nov; 72(Suppl 2):ii1-64.

13 Kwak N, Lee JH, Kim HJ, Kim SA, Yim JJ. New-onset nontuberculous mycobacterial pulmonary disease in bronchiectasis: tracking the clinical and radiographic changes. BMC Pulm Med. 2020 Nov 10;20(1):293. 